\title{
STABILITY IMPROVEMENT OF SUPERCONDUCTING GENERATORS USING ARTIFICIAL INTELLIGENCE-BASED STATIC VAR COMPENSATOR
}

\author{
R. A. F. Saleh \\ Electrical Engineering Dept., Faculty of Engineering, Minoufiya University
}

\begin{abstract}
In this paper, superconducting generator (SCG) stability enhancement via coordinated design of a governor controller (GC) and a static VAR compensator (SVC)-based fuzzy logic stabilizer is investigated. The GC is a conventional lead stabilizer activated by the speed error signal, while the signal produced by the SVC-based stabilizer is based on the SCG speed deviation and acceleration, and on two fuzzy membership functions reflecting few simple control rules. An objective function is defined and the design problem of efficient GC and SVC-based fuzzy stabilizer is formulated as an optimization problem. Particle swarm optimization (PSO) technique is employed to search for optimal parameters of GC and SVC-based stabilizer. Simulation results show that the proposed PSO-tuned control scheme provides good damping to the SCG, and enhances its stability over a range of operating conditions.
\end{abstract}

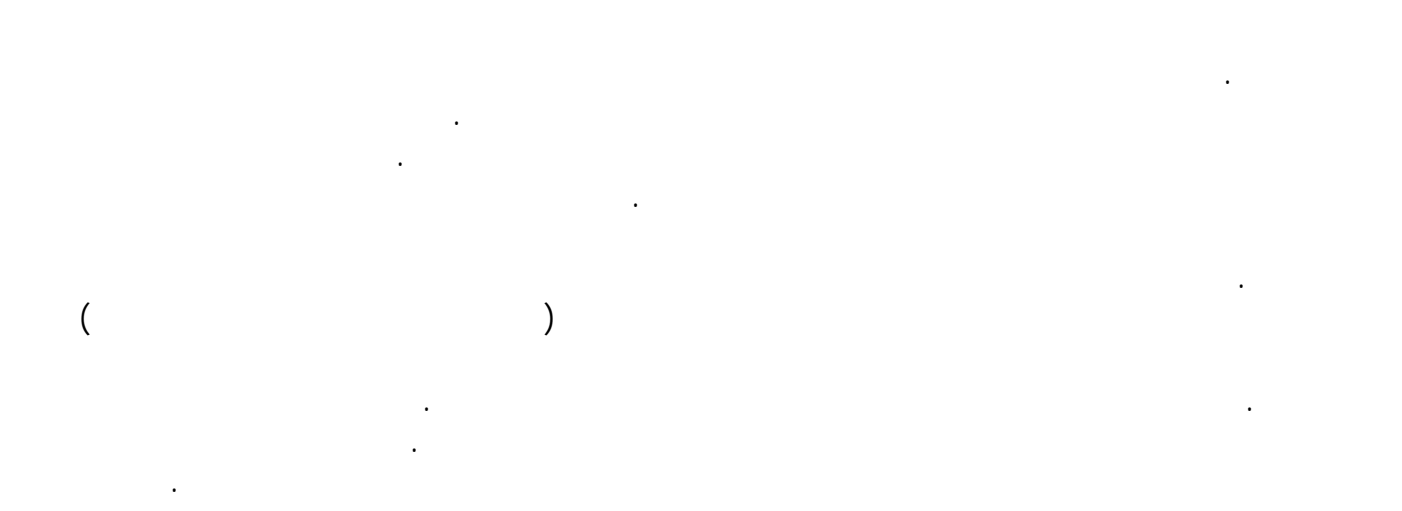

Keywords: Superconducting generator, Transient stability, FACTS, Fuzzy logic control, Particle swarm optimization

\begin{abstract}
1. Introduction
Superconducting generators have several potential advantages such as small size, light weight, high efficiency and increased steady state stability limit [1-2]. The advantages of SCG have drawn more interest in industrial countries since 1970's, such as in USA, UK and Japan where many R\&D projects on SCGs have been conducted at utility companies, power plant manufacturers and other organization toward a 200 MW class pilot-machine [3-7]. Despite these advantages, SCG field winding has an extremely large time constant. The excitation system is therefore not able to change quickly the field current to meet the grid requirements under transient
\end{abstract}

conditions. SCG is also characterized by low inertia and low inherent damping, each of which adversely affects the machine stability when connected to a power system. Inevitably, governor control becomes the feasible technique to enhance stability of superconducting generators. The availability of electro-hydraulic governors and fast operation of steam valves has now made it possible to obtain very fast turbine response. Research works reported in Ref. [8-9] have shown that SCG stability can be improved by introducing a phase advance network in the governor feedback loop, activated by the speed error signal. 
Recently, the flexible AC transmission systems (FACTS) have been introduced, in which various power electronics-based controllers are used to maximize the utilization of transmission assets efficiently and reliably [10-11]. In addition, FACTS devices regulate power flow and, through rapid control actions, can mitigate low frequency oscillations and enhance power system stability [1213].

On the other hand, fuzzy logic stabilizers have appeared as a viable alternative to the conventional stabilizers for enhancing power system stability [1415]. Control scheme based on fuzzy logic is important to consider in view of its potentially lower computational burden and flexible reconstruction. The application of fuzzy control techniques appears to be particularly useful whenever the system to be controlled is complex and has uncertainty and imprecision. These properties certainly apply to power systems incorporating superconducting generators.

Early investigation on the dynamic performance of a SCG when equipped with a static VAR compensator at its terminal was reported in Ref. [16]. In that study, the stabilizing signal was not optimized. Moreover, the governor role in damping the machine oscillation was not considered. However, some efforts have been made towards stability enhancement of SCG using coordinated governor controller and FACTS device-based conventional stabilizer [17]. The conventional stabilizer parameters are fixed to ensure optimum performance at a specific operating point. However, because of the high nonlinearity of the machine/power system combination, the stabilizer's performance becomes lower when the system operating condition moves significantly away from the specific point. Therefore, there should be a degree of robustness in the conventional stabilizer to be able to stabilize the system over a wide range of operating conditions.

In this paper, enhancement of SCG stability using coordinated design of a static VAR compensator (SVC)-based fuzzy stabilizer and a governor controller (GC) is studied. The coordinated optimal parameters of SVC-based fuzzy stabilizer and GC are sought by utilizing the PSO technique [18]. Incorporation of PSO into stabilizer design adds an intelligent dimension to the stabilizer, and reduces the time consumed in the design process. Various non-linear simulation studies are carried out to investigate the effectiveness of the proposed scheme.

\section{System Description}

The system considered in this study is a SCG connected to an infinite bus power system as shown in Fig. 1. The SCG has superconducting field windings in the rotor, surrounded by two separate screens. The inner screen, which has a relatively long time constant, shields the superconducting field windings from external, time varying magnetic fields. The outer screen serves as a damper and has a substantially shorter time constant than that of the inner screen [19]. The SCG is driven by a three-stage steam turbine with reheat between the high pressure and intermediate pressure stages. The turbine is controlled by fast acting electro-hydraulic governors fitted to the main and interceptor valves, which are working in unison. The system is equipped with a governor controller and a SVC at the terminal of the SCG. The exciter voltage, $U_{e}$, of the SCG is kept constant during transients.

\section{Mathematical Model}

The mathematical models for SCG, turbine and governor are shown below, while the parameter values and physical constraints are given in the Appendix.

\subsection{Superconducting generator model}

Based on Park's $d-q$ axis representation, seven nonlinear differential equations are used to represent the mathematical model of the SCG's electric circuits. These equations along with the mechanical equations of motion give the flux linkage model of the SCG [9] as follows:

$$
\begin{aligned}
& p \psi_{d}=\omega_{o}\left[V_{d}+i_{d} R_{a}+\psi_{q}\right]+\psi_{q} \omega \\
& p \psi_{q}=\omega_{o}\left[V_{q}+i_{q} R_{a}-\psi_{d}\right]-\psi_{d} \omega \\
& p \psi_{D 1}=-\omega_{o} i_{D 1} R_{D 1} \\
& p \psi_{Q 1}=-\omega_{o} i_{Q 1} R_{Q 1} \\
& p \psi_{D 2}=-\omega_{o} i_{D 2} R_{D 2} \\
& p \psi_{Q 2}=-\omega_{o} i_{Q 2} R_{Q 2} \\
& p \psi_{f}=\omega_{o}\left[V_{f}-i_{f} R_{f}\right] \\
& p \delta=\omega \\
& p \omega=\frac{\omega_{o}}{2 H}\left[T_{m}-T_{e}\right] \\
& T_{e}=\psi_{d} i_{q}-\psi_{q} i_{d}
\end{aligned}
$$




\subsection{Turbine and governor model}

The mathematical model of the turbine and governor system is represented by six non-linear differential equations [18] as follows:

$$
\begin{aligned}
& p Y_{H P}=\left(G_{M} P_{o}-Y_{H P}\right) / \tau_{H P} \\
& p Y_{R H}=\left(Y_{H P}-Y_{R H}\right) / \tau_{R H} \\
& p Y_{I P}=\left(G_{I} Y_{R H}-Y_{I P}\right) / \tau_{I P} \\
& p Y_{L P}=\left(Y_{I P}-Y_{L P}\right) / \tau_{L P} \\
& p G_{M}=\left(U_{g}-G_{M}\right) / \tau_{G M}
\end{aligned}
$$

$$
p G_{I}=\left(U_{g}-G_{I}\right) / \tau_{G I}
$$

The output mechanical torque is given as:

$$
T_{m}=F_{H P} Y_{H P}+F_{I P} Y_{I P}+F_{L P} Y_{L P}
$$

where:

$$
\begin{array}{ll}
P_{o} & : \text { boiler steam pressure } \\
Y & : \text { output of a turbine or reheat stage } \\
\tau & : \text { time constant of stage } \\
G_{\mathrm{M}}, G_{\mathrm{I}} & : \text { main and interceptor valve positions }
\end{array}
$$

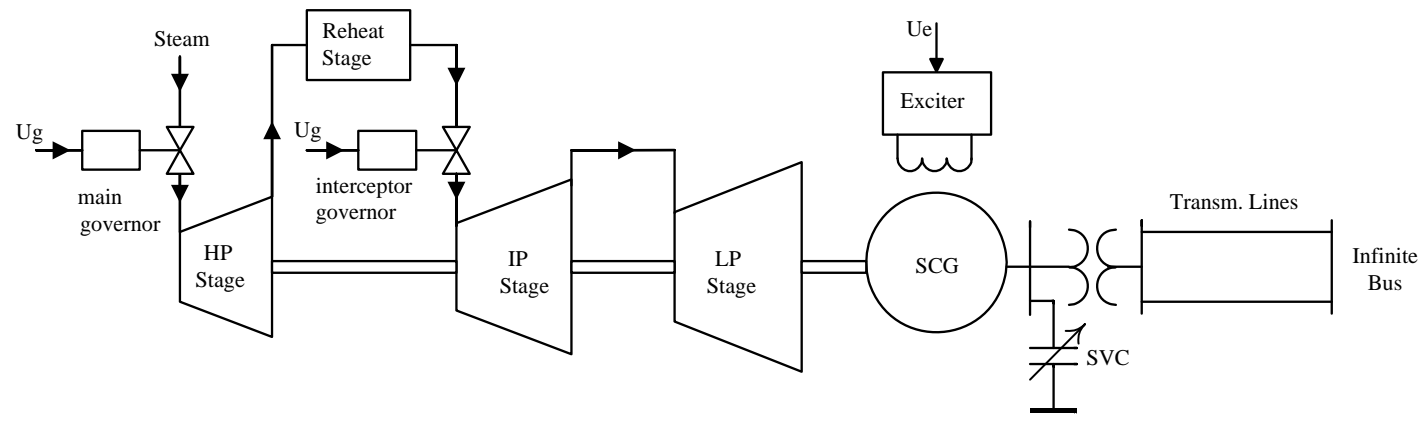

Fig. 1 SCG interconnected to an infinite bus

$F_{H P}, F_{I P}, F_{L P}$ : fractional contributions of turbine stages into $T_{m}$

$U_{g} \quad$ : governor actuating signal

The main and interceptor valves are conventionally actuated by a normalized speed error signal incorporating a droop, typically $4 \%$. Constraints are imposed on valve positions and rates of movement. The rate constraint is based on complete opening or closing time for the valves of $150 \mathrm{~ms}$. The rate limits correspond to the fastest valve operation reportedly available in literature [20].

\section{The Proposed Approach}

\subsection{Control objective}

The control objective is to generate two stabilizing signals using the speed error signal. The first control signal is produced via a conventional controller and then introduced into the governor loop of the SCG system as shown in Fig. 2. The control signal, $u$, generated by the conventional controller is given as:

$$
u=G_{s} \cdot \frac{\left(1+T_{1} s\right)}{\left(1+T_{2} s\right)} \cdot \omega
$$

where $\omega$ is the speed error signal, $G_{s}, T_{1}$ and $T_{2}$ are the controller parameters, which have to be designed properly to achieve a satisfactory performance.

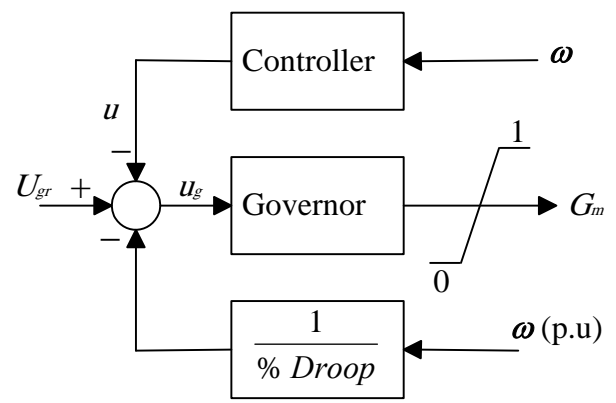

Fig.2 The governor control system

The second signal is produced via a SVC-based fuzzy stabilizer. The two stabilizing signals are coordinated to enhance the damping of the rotor oscillations after disturbances, and hence to improve the transient and dynamic performance of the system.

\subsection{SVC-based fuzzy stabilizer}

The block diagram of an SVC with a fuzzy stabilizer is shown in Fig. 3. Functionality, the SVC is thought of as an adjustable shunt susceptance that can be varied with sufficient rapidity. Elaborated model for SVC can be seen in Ref. [21]. However, the susceptance, $B$, of the SVC can simply be expressed as:

$$
p B=\left(K_{s v c}\left(B_{r e f}+u_{S v C}\right)-B\right) / T_{s v c}
$$

where $K_{s v c}$ and $T_{s v c}$ are the gain and time constant of the SVC. $B_{r e f}$ is the reference susceptance of the SVC 
and $u_{S V C}$ is the stabilizing signal generated by the fuzzy stabilizer installed in the feedback loop of the SVC shown in Fig. 3.

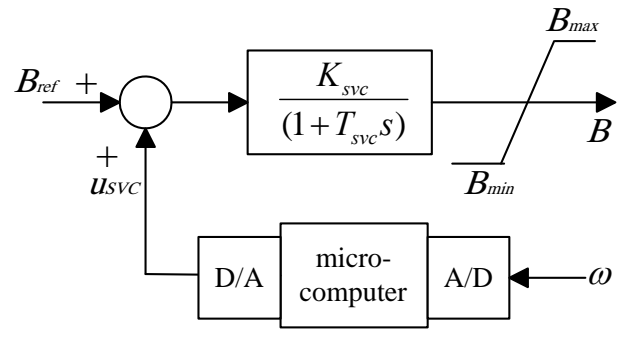

Fig.3 SVC with digital (fuzzy) stabilizer

The signal $u_{S V C}$ is synthesized using fuzzy logic as follows. Fuzzy logic is the logic underlying modes of reasoning which are approximate rather than exact. Thus it is closer to human reasoning and real world than formal logic.

The SCG condition is defined at every sampling time, $k T_{s}$, in terms of its speed deviation and scaled acceleration, $\quad\left[\omega(k), \quad F^{*} d \omega(k) / d t\right]$, where $d \omega(k) / d t=[\omega(k)-\omega(k-1)] / T_{\mathrm{s}}, T_{s}$ is the sampling interval and $F$ is a predefined scaling factor. This condition represents a certain point, $\mathrm{Z}$, in the $[\omega(k)$, $\left.F^{*} d \omega(k) / d t\right]$ phase plane as shown in Fig. 4. The polar displacement $\mathrm{D}(k)$ of this point from the origin, and the corresponding angle $\theta(k)$ are computed as:

$$
\begin{gathered}
D(k)=\left[(\omega(k))^{2}+\left(F^{*} \dot{\omega}(k)\right)^{2}\right]^{0.5} \\
\theta(k)=\tan ^{-1}\left(F^{*} \dot{\omega}(k) / \omega(k)\right)
\end{gathered}
$$

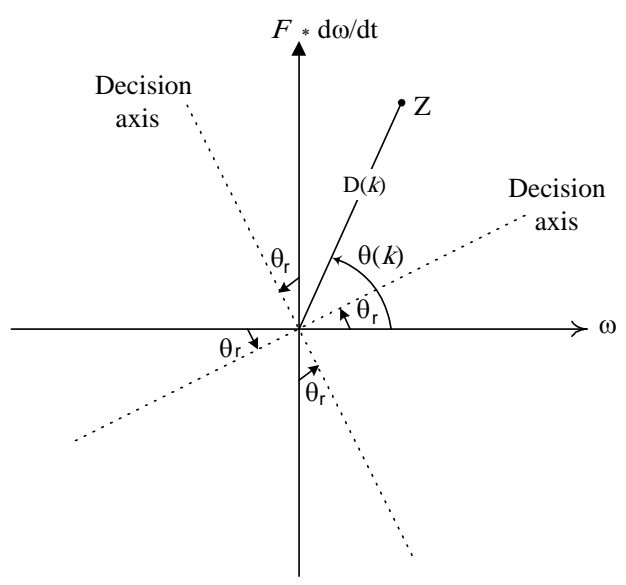

Fig.4 Definition of SCG condition in the phase plane

The phase plane is divided into four quadrants. Each quadrant has simple control rules according to the degree of deceleration and/or acceleration control required to restore the machine condition, after the disturbance, to the origin of the phase plane as soon as possible with an acceptable performance.
Generally, deceleration control and hence a positive control signal, is only required when the SCG status locates in a certain quadrant (say the first quadrant). Acceleration control and hence a negative control signal is required only when the SCG status lies in the opposite quadrant (i.e. the third quadrant). Decreasing (or increasing) deceleration and increasing (or decreasing) acceleration as well are required when the SCG status lies in the other two (i.e. the second and the fourth) quadrants. Two fuzzy membership functions, $N(\theta)$, shown in Fig. 5, associated with the desired deceleration, and $P(\theta)$ associated with the desired acceleration, are defined in terms of the polar angle, $\theta$, defined by equation (21) to reflect the actions of the control rules. The defining relations for $N(\theta)$ and $P(\theta)$ are:

$$
\begin{aligned}
& N(\theta)= \begin{cases}1 & \text { for } \theta \leq \theta_{\mathrm{i}} \\
\left(\theta_{1}-\theta\right) /\left(\theta_{1}-\theta_{i}\right) & \text { for } \theta_{i}<\theta \leq \theta_{1} \\
0 & \text { for } \theta_{1}<\theta \leq \theta_{2} \\
\left(\theta-\theta_{2}\right) /\left(\theta_{f}-\theta_{2}\right) & \text { for } \theta_{2}<\theta \leq \theta_{\mathrm{f}}\end{cases} \\
& P(\theta)=1-N(\theta) \quad \text { for all } \theta
\end{aligned}
$$
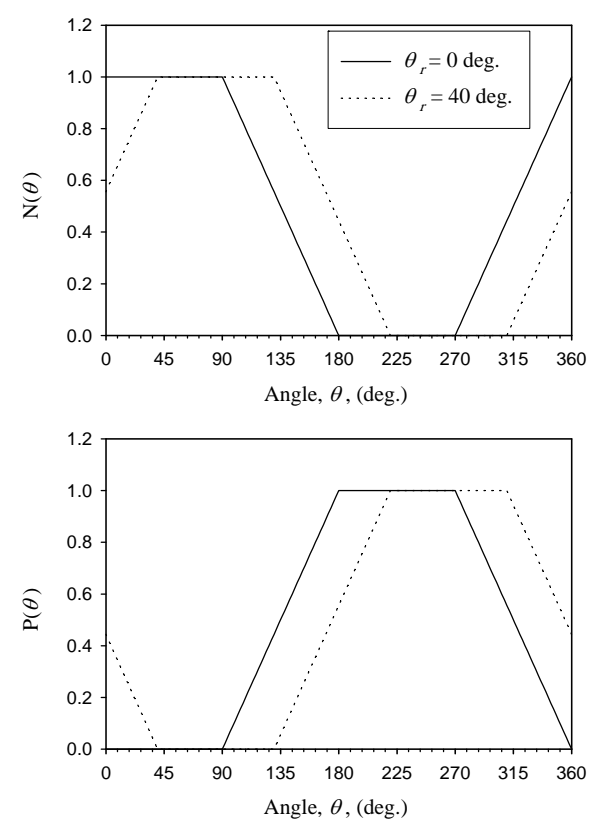

Fig.5 Proposed fuzzy membership functions

The angles $\theta_{i}, \theta_{1}, \theta_{2}$, and $\theta_{f}$ are normally fixed at 90 , 180, 270 and 360 degrees respectively with excitation control of conventional generators [22]. The fuzzy membership functions described by equations (22) and (23) can be portrayed in terms of a pair of what can be termed "decision axes", shown in Fig. 4, on the phase plane. It was found by the present investigator that, for best results, the angles $\theta_{i}, \theta_{1}, \theta_{2}$, and $\theta_{f}$ should again progress in 90 degree 
steps, but that an offset angle $\theta_{r}$ between the phase plane axis set (i.e. the $\omega(k)$ and $F^{*} d \omega(k) / d t$ axes) and the decision axis set (i.e. quadrant boundaries) should be introduced as shown in Fig. 4. This offset angle $\theta_{r}$, which can be regarded as a new tuning parameter, is hence introduced when designing the fuzzy logic-based stabilizer. It specifies the best location for each quadrant with its particular control rules on the phase plane. In effect, the offset angle $\theta_{r}$ rotates the decision axis set anti-clockwise until the minimum of a predefined performance index is obtained.

This has the effect in turn of changing the final shapes of fuzzy membership functions over the whole universe of discourse, i.e. another set of control rules is generated according to the degree of rotation $\theta_{r}$. The resulting two membership functions then lead to a governor control signal, $u_{S V C}(k)$, given by:

$$
u_{S V C}(k)=\mathrm{G}(k)[N(\theta(k))-P(\theta(k))] u_{\max }
$$

where $\mathrm{G}(k)$ is the gain whose value is defined as:

$$
\begin{aligned}
& \mathrm{G}(k)=\mathrm{D}(k) / \mathrm{D}_{\mathrm{r}} \quad \text { for } \mathrm{D}(k)<\mathrm{D}_{\mathrm{r}} \\
& \mathrm{G}(k)=1 \quad \text { for } \mathrm{D}(k) \geq \mathrm{D}_{\mathrm{r}}
\end{aligned}
$$

The parameter $D_{r}$ is a set value of polar displacement at which the gain is required to saturate at unity. However, the implementation of the above SVCbased fuzzy stabilizer requires the following steps in each sampling time:

Step1: SCG speed deviation, $\omega(k)$, is sampled and the scaled acceleration, $F^{*} d \omega(k) / d t$, is computed.

Step2: $\mathrm{D}(k)$ and $\theta(k)$ are determined using equations $(20,21)$.

Step3: Values of both fuzzy membership functions, $N(\theta)$ and $P(\theta)$, are calculated.

Step4: The control signal $u_{S V C}(k)$ is determined using equation (24).

Both of $u$ and $u_{S V C}$ has upper and lower limits, i.e.

$$
u_{\min } \leq\left(u, u_{S V C}\right) \leq u_{\max }
$$

\section{Stabilizer Parameters Selection Using PSO}

Recently, a heuristic search method called particle swarm optimization (PSO) has been introduced [23]. PSO is characterized as a simple concept, easy to implement, and computationally efficient. Theses features make PSO technique able to accomplish the same goal as GA optimization in a new and faster way. A number of very recent successful applications of PSO on various power system problems have been reported in literature [18].

The tuning parameters of the proposed coordinated control scheme are $F, \mathrm{D}_{\mathrm{r}}$ and $\theta_{r}$ for the SVC-based fuzzy stabilizer and $G_{\mathrm{s}}, T_{1}$ and $T_{2}$ for the governor controller (GC). $u_{\max }$ (the maximum size of the control signal) is a pre-specified, constant parameter. For optimal settings of the tuning parameters, the quadratic performance index, $J$, defined by equation (28) is also used. For convenience, it is recalled here:

$$
J=\sum_{k=1}^{N}\left\{[k T . \omega(k)]^{2}+[\Delta \delta(k)]^{2}+\left[\Delta G_{M}\right]^{2}\right\}
$$

Where $\Delta \delta(k)$ and $\Delta G_{M}(k)$ are the deviations of the rotor angle and the governor valve position respectively from their steady state values. In addition, $N$ denotes the total number of time steps. This index is chosen because a low value of it reflects small settling time, small steady state error, and small overshoots in each of rotor speed, rotor angle, and valve position. The tuning parameters are selected to minimize this performance index subject to the following constraints:

$$
\begin{aligned}
& F_{\text {min }} \leq F \leq F_{\text {max }} \\
& D_{r, \text { min }} \leq D_{r} \leq D_{r, \text { max }} \\
& \theta_{r, \text { min }} \leq \theta_{r} \leq \theta_{r, \text { max }} \\
& G_{S, \text { min }} \leq G_{S} \leq G_{S, \text { max }} \\
& T_{1, \text { min }} \leq T_{1} \leq T_{1, \text { max }} \\
& T_{2, \text { min }} \leq T_{2} \leq T_{2, \text { max }}
\end{aligned}
$$

The digital simulation for the SCG system is used in conjunction with the PSO process, which is then used to search for the optimal set of the tuning parameters, which minimizes the performance index chosen. PSO itself has a number of parameters to be properly specified. The main PSO parameters are the initial inertia weight, $w^{0}$, and the maximum allowable velocity, $V_{\max }$. These parameters and other PSO parameters are set as in [17].

\section{Simulation Results}

A number of simulation studies has been performed to investigate the effectiveness of the proposed SVCbased fuzzy stabilizer in improving stability of the SCG under study. The performance index was evaluated, in all cases, in response to a three-phase to ground fault of $120-\mathrm{ms}$ duration with the operating point $\left(P_{t}=0.8\right.$ p.u, $Q_{t}=0.6$ p.u). Variation of the performance index $J$ with the number of iterations is shown in Fig. 6 in two cases. In the first case, the optimal set of $\left(F, \mathrm{D}_{\mathrm{r}}, \theta_{r}\right)$ for the SVC-based fuzzy stabilizer was searched for; considering governor controller (GC) with $G_{\mathrm{s}}=0.1 T_{1}=0.5 \mathrm{~s}$ and $T_{2}=0.01 \mathrm{~s}$ [24]. In the second case, coordinated design for best combination of $\left(F, \mathrm{D}_{\mathrm{r}}, \theta_{r}\right)$ for the SVC-based fuzzy stabilizer and $\left(G_{\mathrm{s}}, T_{1}, T_{2}\right)$ for the GC was sought. From this figure, it is concluded that the coordinated design (using PSO) for the SVC-based fuzzy 
stabilizer and the GC results in more improvement in the SCG performance.

The optimal coordinated values selected by PSO for $\left(F, \mathrm{D}_{\mathrm{r}}, \theta_{r}\right)$ and $\left(G_{\mathrm{s}}, T_{1}, T_{2}\right)$ are $\left(0.028,0.923,3.84^{\circ}\right)$ and $(0.061,1,0.01)$ respectively. Performance of the SCG system with the optimally designed control scheme following a 3-phase short circuit fault, at [ $\left(P_{t}\right.$, $\left.Q_{t}\right)=(0.8,0.6),(0.7,-0.2)$ p.u] is shown in Fig. 7 and Fig. 8. Figures 9 and 10 show the system response to a temporary (100-ms long) $10 \%$ step increase in the governor set point $\left(\mathrm{U}_{\mathrm{gr}}\right)$ at the considered loading conditions.

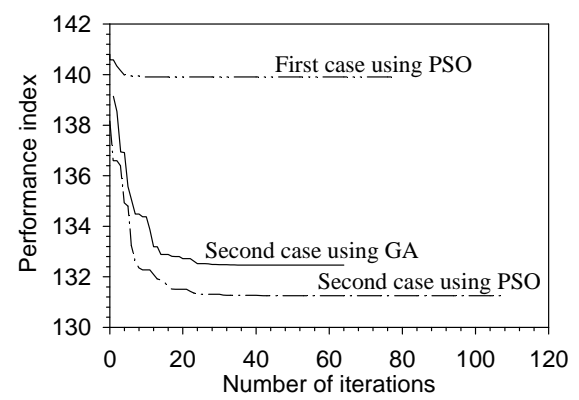

Fig. 6 Convergence of performance index with iterations using PSO \& GA
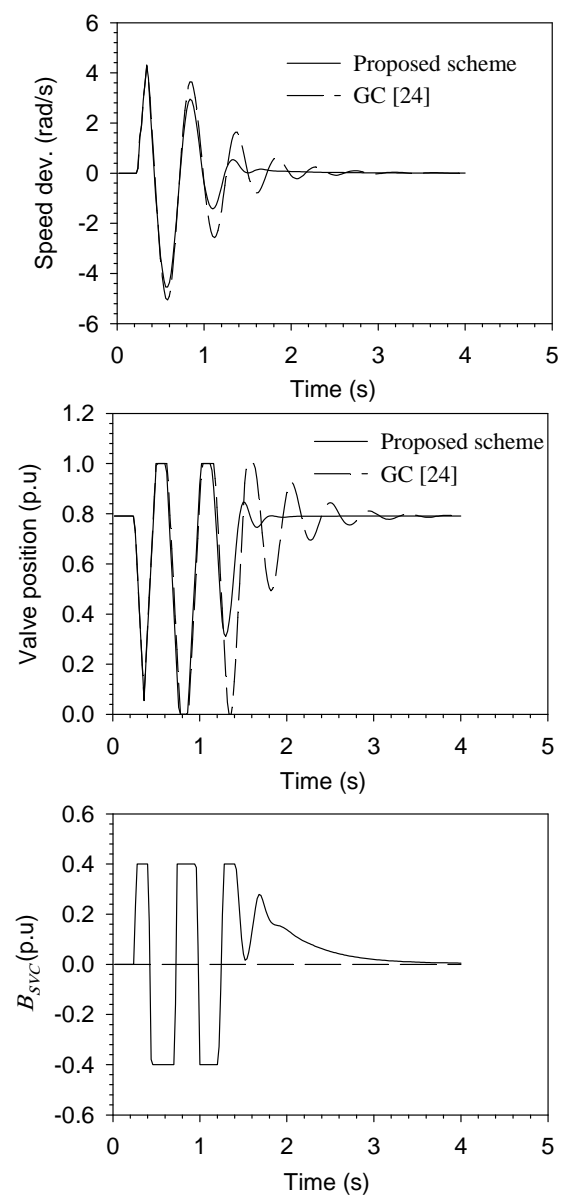

Fig. 7 Response to a 3-phase $\mathrm{SC}$ at $\mathrm{P}_{\mathrm{t}}=0.8 \mathrm{pu}, \mathrm{Q}_{\mathrm{t}}=0.6$ $\mathrm{pu}$
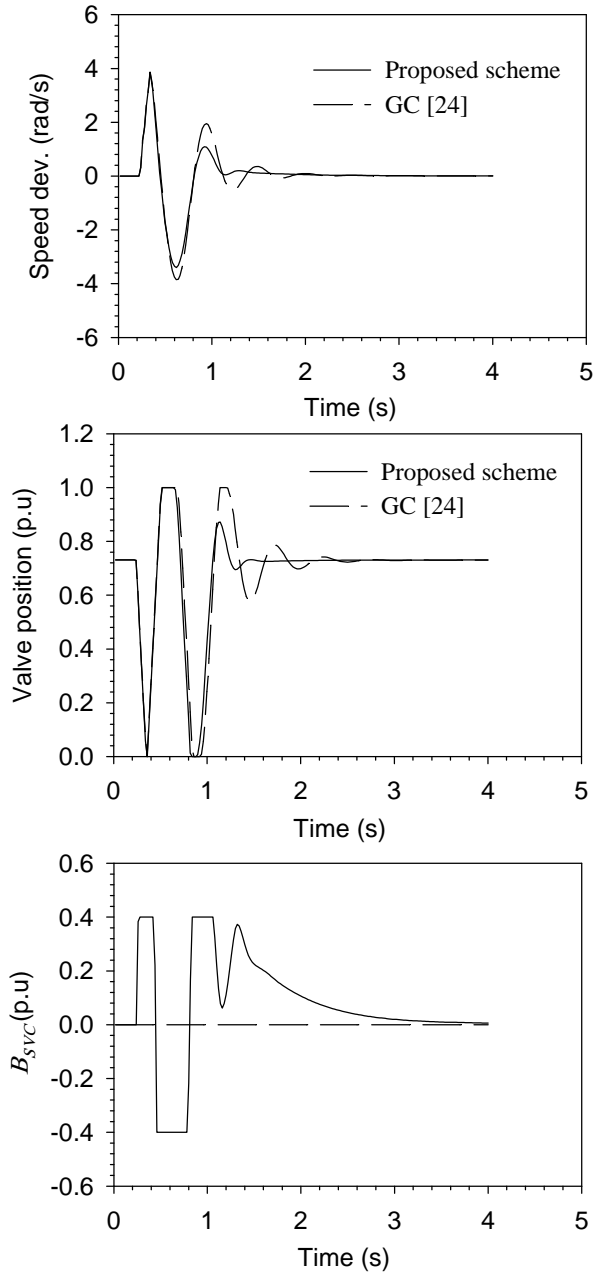

Fig. 8 Response to a 3-phase SC at $\mathrm{P}_{\mathrm{t}}=0.7 \mathrm{pu}, \mathrm{Q}_{\mathrm{t}}=-0.2 \mathrm{pu}$

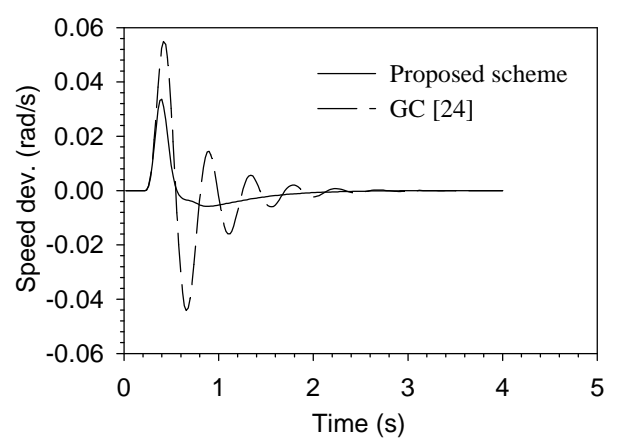

Fig. 9 Response to a $10 \%$ pulse in $\mathrm{U}_{\mathrm{gr}}$ at $\mathrm{P}_{\mathrm{t}}=0.8 \mathrm{pu}, \mathrm{Q}_{\mathrm{t}}=0.6 \mathrm{pu}$ 


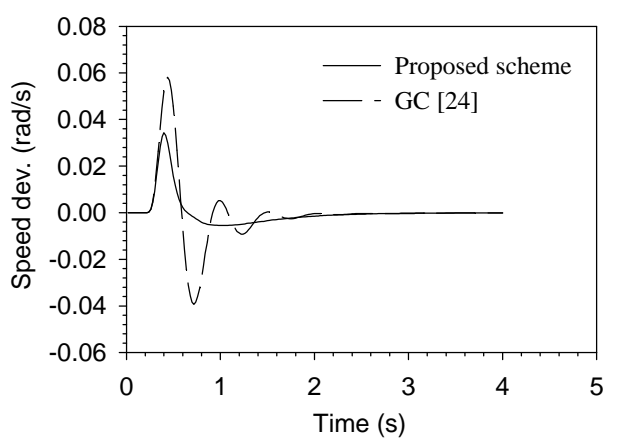

Fig. 10 Response to a $10 \%$ pulse in $\mathrm{U}_{\mathrm{gr}}$ at $\mathrm{P}_{\mathrm{t}}=0.7 \mathrm{pu}, \mathrm{Q}_{\mathrm{t}}=-0.2 \mathrm{pu}$

The results show that the proposed control scheme results in a significant improvement in the SCG transient performance (after major and minor disturbances) and a considerable reduction in the rotor oscillations with acceptable valve movements at various loading conditions. The corresponding results of damping and synchronizing torques analysis, using the technique described in [17], are shown in Table 1, in comparison with other schemes (viz. SVC with GC [17], and GC [24] only).

Table 1: Comparison of the proposed scheme and other schemes

\begin{tabular}{|l|c|c|c|c|c|}
\hline \multicolumn{1}{|c|}{$\left(P_{t}, Q_{t}\right)$ p.u } & \multicolumn{3}{|c|}{$(0.8,0.6)$} & \multicolumn{2}{c|}{$(0.7,-0.2)$} \\
\hline & $J$ & $K_{d}$ & $k_{s}$ & $K_{d}$ & $k_{s}$ \\
\hline $\begin{array}{l}\text { Proposed } \\
\text { scheme }\end{array}$ & 131.25 & 0.25 & 1.996 & 0.242 & 1.223 \\
\hline $\begin{array}{l}\text { SVC with } \\
\text { GC [17] }\end{array}$ & 130.2 & 0.231 & 1.941 & 0.212 & 1.184 \\
\hline GC [24] & 261.7 & 0.014 & 2.011 & 0.016 & 1.251 \\
\hline
\end{tabular}

From this table, it can be concluded that the proposed scheme outperforms the other considered schemes at the operating points studied. It provides the SCG system with the highest possible degree of damping while keeping the synchronizing torque at a high level.

\section{Conclusion}

This paper investigated the application of one of FACTS devices for stability enhancement of superconducting generators. An approach was proposed for the design of a static VAR compensator-based fuzzy stabilizer in coordination with a governor controller to provide more damping to mechanical oscillations of the SCG studied. A performance index was defined and the PSO technique was used to select the optimal parameters of both SVC-based fuzzy stabilizer and GC. Simulation results show the effectiveness of the proposed control scheme in damping the rotor oscillations, and enhancing the SCG stability over a range of operating conditions and various disturbances. Analysis of damping and synchronizing torques was used to provide another quantitative assessment of the SCG performance with the designed SVC-based fuzzy stabilizer and GC. Results of non-linear simulation studies show the effectiveness of the proposed approach in enhancing SCG stability.

\section{References}

[1] N. Maki, K. Yamaguchi, M. Takahashi, and R. Shiobara, "Development of super-conducting AC generator,” IEEE Trans. on Magnetics, Vol. 24, No. 2, pp. 792-795, 1988.

[2] T. Nitta, Y. Shirai, T. Kawauchi, T. Okada, and Y. Ogawa, "Transient stability limit issues at threephase short-circuit in parallel running of both a superconducting generator and a conventional one", Electrical Engineering in Japan, Vol. 115, No. 6, pp. 62-70, 1995.

[3] K. Ueda, R. Shiobara, M. Takahashi, and T. Ageta, "Measurement and analysis of $70 \mathrm{MW}$ superconducting generator constants", IEEE Trans. on Applied Superconductivity, Vol.9, No.2, pp.11931196, 1999.

[4] H. Tsukiji, T. Hoshino, and I. Muta, "Output power limit of 200 MW class brushless superconducting generator excited with magnetic flux pump” IEEE Trans. on Applied Super-conductivity, Vol. 11, No.1, pp.2335-2338, 2001.

[5] http://events.energetics.com/wire05/, K. Amm, "100 MVA HTS Generator Development Update", DOE HTS Wire Workshop, January 19-20, 2005.

[6] M. Maki, "Design study of high-temperature superconducting generators for wind power systems", 2008, Journal of Physics: Conf. Ser. 97012155 (6pp) [7] K. F. Goddard, B. Lukasik, and J.K. Sykulski, "Alternative Designs of High-Temperature Superconducting Synchronous Generators", IEEE Trans. on Applied Superconductivity, Vol. 19, No.6, pp. 3805-3811, 2010.

[8] M. A. A. S. Alyan and Y. H. Rahim, "The role of governor control in transient stability of superconducting turbo-generators," IEEE Trans. on EC, Vol. 2, No. 1, pp. 38-46, 1987.

[9] S.M. Osheba, M.A.A.S. Alyan, and Y.H.A. Rahim, "Comparison of transient performance of superconducting and conventional generators in a multi-machine system”, IEE Proc., Pt. C, Vol. 135, No.5, pp. 389-395, 1988.

[10] N.G. Hingorani, "High power electronics and flexible AC transmission system", IEEE Power Engineering Review, July 1988. 
[11] A. Edris, "FACTS technology development: an update", IEEE Power Engineering Review, pp.4-9, March 2000.

[12] M. Noroozian and G. Anderson, "Damping of power system oscillations by use of controllable components", IEEE Trans. PWRD, Vol.9, No.4, pp.2046-2054, 1994.

[13] M. A. Abido and Y. L. Abdel-Magid, "Coordinated design of a PSS and an SVC-based controller to enhance power system stability", International Journal of Electrical Power and Energy Systems, Vol.25, No.9, pp. 695-704, 2003.

[14] T. Hiyama, "Robustness of fuzzy logic power system stabilizers applied to multi-machine power system”, IEEE Trans. on EC, V.9, N.3, pp. 451-459, 1994.

[15] A.M.D. Ferreira, J.A.L. Barreiros, J.R. Brito-deSouza, "Fuzzy power system stabilizer including a fuzzy PI controller”, UKACC International Conference on Control, V.1, pp. 865-870, Sept. 1998. [16] R.M. Mathur, P.K. Dash and A.E. Hammad, "Transient and small signal stability of a superconducting turbo-generator operating with thyristor controlled static compensator", IEEE Trans. on PAS, Vol.98, No.6, pp.1937-1946, 1979.

[17] R.A.F. Saleh, "A particle swarm-aided approach for stability enhancement of a superconducting generator using an SVC-based stabilizer", Engineering Research Journal, Vol. 34, No. 2, pp. 103-110, 2011.

[18] A. A. Abou El-Ela, T. Fetouh, M. A. Bishr and R.A.F. Saleh, "Power systems operation using particle swarm optimization technique”, Electric Power Systems Research 78, pp. 1906-1913, 2008.

[19] P. J. Lawrenson, T. J. E. Miller, J. M. Stephenson, and A. H. M. S. Ula, "Damping and screening in the synchronous superconducting generator,” Proc. IEE, Vol. 123, No. 8, pp. 787-794, 1976.

[20] B.W. Hogg, "Representation and control of turbogenerators in electric power systems", Chapter 5 in 'Modelling of dynamical systems', Vol.2, Peter Peregrinus Ltd., 1981.

[21] M. Pavella, and P.G. Murthy, "Transient stability of power systems: theory and practice”, John Wiley \& Sons, 1st edition, 1994.

[22] T. Hiyama, K. Miyazaki, and H. Satoh, “A fuzzy logic excitation systems for stability enhancement of power systems with multi-mode oscillations”, IEEE Trans. on Energy Conversion, V.11, N.2, pp. 449454, 1996.

[23] R. Eberhart, and Y. Shi, "Particle swarm optimization: developments, applications and resources”, Proc. of the 2001 Congress on Evolutionary Computation, Vol. 1, pp. 81-86, 2001.
[24] G.A. Morsy, A. Kinawy, and S.M. Osheba, "Frequency domain analysis of a superconducting generator”, Electric Power Systems Research, No. 30, pp. 107-113, 1994.

\section{Appendix}

The parameters of the SCG system used in this study (inductance and resistance values in p.u; time constants in seconds) are [8, 9]:

Superconducting generator parameters:

$L_{f}=0.541, L_{d}=L_{q}=0.5435, L_{D I}=L_{Q I}=0.2567$,

$L_{D 2}=L_{Q 2}=0.4225$

$L_{f d}=L_{f D 1}=L_{d D 1}=L_{d D 2}=L_{D 1 D 2}=0.237$

$L_{f D 2}=0.3898, L_{q Q 1}=L_{q Q 2}=L_{Q 1 Q 2}=0.237$

$\tau_{\mathrm{f}}=750, R_{d}=R_{q}=0.003$

$R_{D I}=R_{Q 1}=0.01008, R_{D 2}=R_{Q 2}=0.00134$

$H=3 \mathrm{~kW} . \mathrm{s} / \mathrm{kVA}$

Transformer and transmission line parameters:

$X_{T}=0.15, R_{T}=0.003, X_{L}=0.05, R_{L}=0.005$

Turbine and governor parameters:

$\tau_{G M}=\tau_{G I}=0.1, \tau_{H P}=0.1, \tau_{R H}=10$,

$\tau_{I P}=\tau_{L P}=0.3, P_{o}=1.2$ p.u.

$F_{H P}=0.26, F_{I P}=0.42, F_{L P}=0.32$

Valve position and movement constraints are defined by:

$0 \leq\left(G_{M}, G_{I}\right) \leq 1$ and $-6.7 \leq\left(p G_{M}, p G_{I}\right) \leq 6.7$

\section{Acknowledgment}

The author would like to acknowledge the support of Scientific Research Deanship, Qassim University, Saudi Arabia. Research Project No. SR-D-010-326. 